(Aus der Universitätsfrauenklinik und dem Physiologischen Institut Kiel.)

\title{
Untersuchungen über die Senkungsgeschwindigkeit der roten Blutkörperchen.
}

\author{
1. Mitteilung. \\ Beobachtungen am menschlichen Blut. \\ Von \\ Priv.-Doz. Dr. Georg Linzenmeier, \\ Assistent der Univ.-Frauenklinik. \\ (Eingegangen am 5. Februar 1920.)
}

Der Schwede Fåhraeus ${ }^{1}$ ) hat uns 1918 mit der Tatsache bekannt gemacht, daß die roten Blutkörperchen bei verschiedenen Menschen und bei besonderen physiologischen und pathologischen Zuständen verschieden rasch zu Boden sinken. Der Unterschied im Fallen der B. K. im Blute einer normalen Frau und einer schwangeren Frau ist so groß, daß Fåhraeus in der Senkungsbeschleunigung der Blutkörperchen ein Schwangerschaftsdiagnostikum erblickte ${ }^{2}$ ).

In seinen Untersuchungen über die Ursachen der Instabilität der B. K.-Suspensionen war Fåhrae us zu der Erkenntnis gelangt, daß die rasche Senkung der Blutkörperchen in erster Linie Folge einer Hämagglutination ist. Durch Verklumpung der B. K. und größere Aggregatbildungen wird ihre Oberfläche verkleinert und damit aus rein physikalischen Gründen ein rascheres Fallen bedingt.

Bis zu dieser Erkenntnis waren auch schon die alten Hämatologen, wie besonders H. Nasse, Johannes Müller ${ }^{3}$ ) und andere gelangt; ihnen war bereits eine vermehrte Blutkörperchensenkungsgeschwindigkeit in der Schwangerschaft und vor allem bei entzündlichen, fieberhaften Erkrankungen gut bekannt. In neuerer Zeit hat de $\mathrm{Haan}^{4}$ ) ebenfalls eine vermehrte Geldrollenbildung der B. K. als Ursache für die schnelle Sedimentierung des Pferdeblutes angesehen.

1) Hygiea 1918.

2) Meine klinischen Untersuchungen zu diesem Thema werden im Arch. f. Gynäkol. veröffentlicht.

3) Angaben über die ältere Literatur finden sich in meiner klinischen Arbeit.

$\left.{ }^{4}\right)$ Biochem. Zeitschr. 86. 1918. 


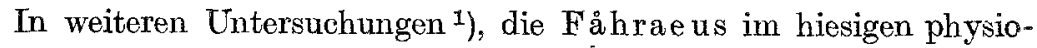
logischen Institut unter Leitung Höbers ausgeführt hat, wurde nun festgestellt, daß die nähere Ursache der Agglutination eine starke Entladung der negativ geladenen B. K. ist. Die Blutkörperchen von. Graviden werden vom Plasma aus mehr oder weniger entladen, dadurch verlieren sie ihre Suspensionsstabilität, agglutinieren und senken sich als größere Aggregate schnell zu Boden.

Wenn wir die Zustände betrachten, bei denen eine vermehrte Senkungsgeschwindigkeit der B. K. stattfindet, so fällt der Parallelismus auf, der mit der Abderhaldenschen Reaktion besteht; bei sämtlichen Zuständen, in denen Abderhalden mit dem Vorkommen von blutfremden Eiweißkörpern im Blut rechnet, ist die Stabilität der Blutkörperchensuspensionen herabgesetzt. Fåhrae us vermutet daher, daß ,die Plasmahaut, die nach Höber als Träger der Grundladung anzusehen ist, ihre Ladung dadurch verliert, daß die sie aufbauenden Eiweißkörper durch Adsorption irgendwie elektrisch neutralisiert werden."

Meine eigenen Untersuchungen, die ich ebenfalls am hiesigen physiologischen Institut unter Höber ausgeführt habe, gehen darauf aus, die Konsequenzen aus dieser Hypothése zu ziehen.

Die erste Frage, die ich mir stellte, war daher: Auf welchent Eigenschaften des Plasmas beruht die entladende Wirkung?

Es war naheliegend zu versuchen, die sen kungsbeschleunigende S u bstanz oder mit einem anderen Worte das fragliche "Agglutinin“ auf irgendeine Weise aus dem Plasma zu entfernen. Ich versuchte zunächst, ob dies durch Ausschütteln des Plasmas mitgeeigneten Adsorbenzien gelingen würde. Wenn die senkungsbeschleunigende Substanz des Plasmas durch Entladung die B. K. agglutiniert, so ist nämlich zu vermuten, daß sie positive Ladung führt, daß sie also durch solche Adsorbenzien zu entfernen sein würde, welche auch sonst positiv geladene Bestandteile aufnehmen, während die Behandlung des Plasmas mit Adsorbenzien, welche negative Teile adsorbieren, die Blutkörperchensenkung nicht verzögern dürfte.

Zur Orientierung über das relative Adsorptionsvermögen für positive Teilchen wurde in einem Vorversuch eine Reihe von Adsorbenzien mit Methylenblaulösung geschüttelt; in dieser ist der basische Bestandteil, das Farbstoffkation, der vornehmlich adsorbierbare Anteil. Das Ergebnis ist in der folgenden Tabelle wiedergegeben.

Danach sind Kaolin, Bolus und Tierkohle geeignet, positive Teilchen aus einer Lösung wegzuadsorbieren; Aluminiumhydroxyd, Cerioxyd, Eisenoxyd und Eisenhydroxyd sind dafür mehr oder weniger ungeeignet; Talkum nimmt eine Mittelstellung ein.

1) Biochem. Zeitschr. 89. 1918. 
$1 \mathrm{~g}$ Adsorbens, $5 \mathrm{ccm}$ Lösung Metbylenblau rektifik. n. Whrlich (Grübler).

Absorbens

1. Aluminium hydroxyd (E. Merck)

2. Cerioxyd (Kahlbaum) . . . .

3. Eisenoxyd (Kahlbaum) . . . .

4. Eisenhydroxyd (Kablbaum). .

5. Talkum (E. Merck) . . . . .

6. Kaolin (Kahlbaum) . . . .

7. Bolus alba (E. Merck) . . . .

8. Tierkohle (E. Merck). . . .
Farbe der Lösung nach dem Schüt. teln mit Methylenblaulösung \begin{tabular}{l|l}
0,0025 molar & 0,0005 molar
\end{tabular} dunkelblau dunkelblau

\begin{tabular}{c|c}
$"$ & $"$ \\
$"$ & $"$ \\
fast farblos & farblos \\
farblos & $"$,
\end{tabular}

"

Zu den Versuchen mit Blut benutzte ich Normalfrauenblut, Nabelschnurblut und Schwangerenblut.

Die Untersuchungtechnik war einfach: das Blut wurde durch Venaepunktio entnommen und, um Gerinnung zu vermeiden, mit $\mathbf{5} \%$ Natriumcitratlösung im Verhältnis 1:9 vermischt. Die Flüssigkeit wurde 40 Minuten zentrifugiert, das Plasma zur Verwendung abgehoben und die B. K. zweimal mit $0,95 \%$ Kochsalzlösung gewaschen. Durch Vorversuche hatte ich festgestellt, daß zwischen Plasma und B. K. die schärfste Grenze auftritt bei einem Mischungsverhältnis von Blutkörperchen zu Plasma wie $1: 3$ oder 2:3. Bei kleineren B. K.-Mengen ist die Trennungsgrenze verwaschen und nicht mit Exaktheit abzulesen. Die Messungen nahm ich an der Plasmahöhe mit Zirkel und Maßstab nach bestimmten Zeitintervallen vor.

Zur Ausschüttelung wurden $2 \mathrm{~cm}$. Citratplasma mit $0,5 \mathrm{~g}$ des Adsorbens in einem Reagenzglas vermischt und $11 / 2$ Stunden in einem langsam gehenden Schüttelapparat liegengelassen. Nach Zentrifugierung wurden zu $0,6 \mathrm{ccm}$ Plasma $0,4 \mathrm{ccm}$ (zweimal gewaschene) B. $\mathrm{K}$. hinzugefügt und dann die Blutkörperehensenkungen beobachtet. Wenn nichts besonders erwähnt ist, sind immer die zum. Plasma gehörigen $\mathbf{B}$. $K$. verwendet.

Von sämtlichen angeführten Versuchen sind mindestens zwei angestellt worden.

$$
\text { A. Tierkoble. }
$$

\section{Normalfrauenblut.}

Versuch vom 29. VII. 1919.

\section{a) unverändert}

nach 13 Min. $7 \mathrm{~mm}$ nach 25 Min. $10 \mathrm{~mm}$ nach 40 Min. $12 \mathrm{~mm}$ b) geschüttelt

$2 \mathrm{~mm}$

$3^{1} / 2 \mathrm{~mm}$ $4^{1 / 2} \mathrm{~mm}$
2. Gravidenblut.

Versuch rom 29. VII. 1919.

a) unverändert

nach 10 Min. $13 \mathrm{~mm}$

nach $20 \mathrm{Min} .17 \mathrm{~mm}$ b) geschiuttelt

$3 \mathrm{~mm}$

$\bar{\partial} \mathrm{mm}$

B. Kaolin.

Versuch vom 29. VII. 1919.

nach 10 Min. $7 \mathrm{~mm}$ nach 20 Min. $10 \mathrm{~mm}$ nach 30 Min. $12 \mathrm{~mm}$ nach 2 Std. $16 \mathrm{~mm}$
$3 \mathrm{~mm}$

$4 \mathrm{~mm}$

$6 \mathrm{~mm}$

$7 \mathrm{~mm}$

Versuch vom 29. VII. 1919.

$14 \mathrm{~mm}$

$15 \mathrm{~mm}$

$16 \mathrm{~mm}$

$16 \mathrm{~mm}$
$5 \mathrm{~mm}$

$8 \mathrm{~mm}$

$10 \mathrm{~mm}$

$12 \mathrm{~mm}$

\section{Bolus alba.}

Versuch vom 29. ViIIt. 1919.

nach 20 Min. $10 \mathrm{~mm}$

vach 1 Std. $15 \mathrm{~mm}$

nach 2 Std. $16 \mathrm{~mm}$
$1 \mathrm{~min}$

$3 \mathrm{~mm}$

$5 \mathrm{~mm}$
Versuch vom 12. IX. 1919.

nach 10 Min. $12 \mathrm{~mm}$ nach 20 Min. $14 \mathrm{~mm}$ nach 1 Std. $17 \mathrm{~mm}$
$2 \mathrm{~mm}$

$4 \mathrm{~mm}$

$8 \mathrm{~mm}$ 
D. T alkum.

Versuch vom 10. X. 1919. nach 20 Min. $4 \mathrm{~mm}$ nach 50 Min. $7 \mathrm{~min}$ nach 4 Std. $14 \mathrm{~mm}$

\section{$4 \mathrm{~mm}$ \\ $6 \mathrm{~mm}$}

$12 \mathrm{~mm}$

Versuch vom 16. X. 1919.

Aus den Protokollen geht ohne Zweifel hervor, daß durch die Ausschüttelung des Plasmas mit den Adsorbenzien für positive Teilchen, Tierkohle, Kaolin und Bolus alba, eine starke Hemmung der B. K. - Senkung hervorgerufen wird, während Talkum nur eine mäßige und wechselnde Wirkung hat. Es ist also das eingetreten, was man nach den Methylenblauausschüttelungen erwarten durfte.

Ganz anders ist der Verlauf bei den Adsorbenzien für negative Teilchen.

Versuch vom 2. X. 1919.

$$
\text { A. Eisenoxyd. }
$$

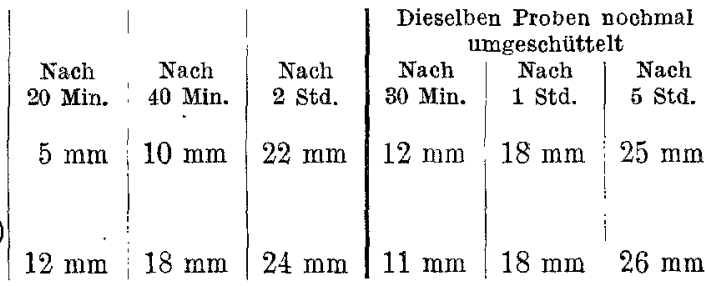

$$
\begin{aligned}
& \text { B. Eisenhydroxyd. }
\end{aligned}
$$

Versuch vom 20. X. 1919.
a) unverändert
b) mit Eisenhydroxyd geschïttelt

nach 30 Min. 6 mus nach 50 Min. $11 \mathrm{~mm}$

$7 \mathrm{~mm}$

$11 \mathrm{~mm}$

nach 1 Std. 50 Min. $18 \mathrm{~mm}$

$19 \mathrm{jnm}$

$$
\text { C. Aluminiumbydrooxyd. }
$$

Versuch vom 4. VIII. 1919.

\section{a) unverändert}

nach $10 \mathrm{Min} .6 \mathrm{~mm}$

nach 20 Min. $11 \mathrm{~mm}$

nach $30 \mathrm{Min} .15 \mathrm{~mm}$ b) geschüttelt

$8 \mathrm{~mm}$

$14 \mathrm{~mm}$

$15 \mathrm{~mm}$

\begin{tabular}{|c|c|c|c|c|c|c|}
\hline & & & & \multicolumn{3}{|c|}{ Neu umgeschüttelt } \\
\hline 0,6 Gravid-Plasma & $\begin{array}{l}\text { Nach } \\
20 \text { Min. }\end{array}$ & $\begin{array}{l}\text { Nach } \\
\text { 35 Min. }\end{array}$ & $\begin{array}{l}\text { Nach } \\
1 \text { Std. }\end{array}$ & $\begin{array}{c}\text { Nach } \\
20 \text { Min. }\end{array}$ & $\begin{array}{l}\text { Nach } \\
\text { 50 Min. }\end{array}$ & $\begin{array}{l}\text { Nach } \\
2 \text { Std. }\end{array}$ \\
\hline 0,4 B. K. . . & $14 \mathrm{~mm}$ & $16 \mathrm{~mm}$ & $17 \mathrm{~mm}$ & $9 \mathrm{~mm}$ & $12 \mathrm{~mm}$ & $25 \mathrm{~mm}$ \\
\hline $\begin{array}{l}0,6 \text { Gravid. - Plasma (mit } \\
\text { Cerioxyd gesch.) } \\
\text { 0,4 B. K. . . . . . . }\end{array}$ & $10 \mathrm{~mm}$ & $13 \mathrm{~mm}$ & $1.5 \mathrm{~mm}$ & $8 \mathrm{~mm}$ & $12 \mathrm{~mm}$ & $15 \mathrm{n}$ \\
\hline
\end{tabular}

D. Cerioxyd.

Versuch vom 12. IX. 19. 
Untersuchungen über die Senkungsgeschwindigkeit der roten Blutkörpërchen. 173

Versuch vom 2. X. 19.

a) unverändert

nach 20 Min. $5 \mathrm{~mm}$

nach 40 Min. $10 \mathrm{~mm}$

nach 2 Std. $22 \mathrm{~mm}$ b) geschüttelt

$11 \mathrm{~mm}$

$16 \mathrm{~mm}$

$20 \mathrm{~mm}$

Um übrigens auszuschließen, daß die Schüttelung an sich und das Abfiltrieren des Adsorbens (Tierkohle) das Citratplasma verändert haben könnte, stellte ich Kontrollversuche an, die ergaben, da.B Schüttelung von 3 Stunden und Filtrierung das Citratplasma unbeeinflußt ließen.

Es erhellt aus diesen Versuchen, daß die Adsorbenzien für negative Teilchen im Gegensatz zu denen für positive Teilchen das Plasma der senkungsbeschleunigenden Substanz nicht berauben; im Gegenteil, es war des öfteren nach dem Schütteln eine vergrößerte Sedimentierungsgeschwindigkeit zu konstatieren, welche aber auffallenderweise nach nochmaligem Umschüttcln der Probe bei abermaliger Sedimentierung nicht mehr zu beobachten war. Dies Phänomen bedarf noch der weiteren Aufklärung. Die Adsorptionsversuche unterstützen also die Schlußfolgerung, daß die senkungsbeschleunigende Substanz ein elektropositiver Körper ist.

Dieser Schluß ließ sich auch durch quantitative Messung der Ladung der B. K., die nach Waschen mit Kochsalzlösung einmal in das unveränderte Plasma und einmal in ausgeschütteltes Plasma eingetragen

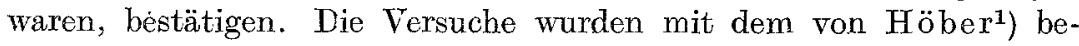
schriebenen Apparat zur Beobachtung der Kataphorese von Zellen ausgeführt. Die Kammer des Apparates wurde mit einer Mischung von isotonischer Rohrzucker- und isotonischer Kochsalzlösung (im Verhältnis $10: 1)$ gefüllt, der Mischung verschiedene Konzentrationen von Lanthannitrat zugesetzt und dann eine Spur Blut darin verrührt. Legt man alsdanin eine genügend.große Spannung an die Enden der Kammer, so kann man unmittelbar darauf im Mikroskop an der Wanderungsrichtung der B. K. erkennen, ob sie negativ oder positiv geladen sind. Das folgende Protokoll belehrt über den Einfluß der Plasmabehandlung wit Kaolin auf die Ladung.

\section{Kataphoreseversuch.}

Gravid.-citrat Blut. Gewaschene Blutkörperchen teils zum Kaolingeschüttelten (1), teils zum unverärderten Citratplasma (2) im Verhältnis $0,4: 0,6$ zugesetzt.

Sedimentierung in 1 : in $30^{\prime} 3 \mathrm{~mm}$, in $120^{\prime} 8 \mathrm{~mm}$, , in $2:$ in $30^{\prime} 12 \mathrm{~mm}$, in $120^{\prime} 16 \mathrm{~mm}$.

1) Arch. f d. ges. Physiol. 101, 607. 1904; 102, 196. 1904. 


\begin{tabular}{|c|c|c|}
\hline \multirow{2}{*}{$\begin{array}{c}\text { Zusatz von } \mathrm{La}\left(\mathrm{NO}_{3}\right)_{3} \\
\text { zum Plasma }\end{array}$} & \multicolumn{2}{|c|}{ Ladung der Blutkörperchen } \\
\hline & 1. im Koalinplasma & 2. im gewöhnlichen Plasma \\
\hline $\begin{array}{l}\mathrm{m} / 2500 \\
\mathrm{~m} / 1500 \\
\mathrm{~m} / 1000 \\
\mathrm{~m} / \mathrm{750} \\
\mathrm{xa} / 500\end{array}$ & $\begin{array}{l}\text { negativ } \\
\text { negativ } \\
\text { vielleicht schwach positiv } \\
\text { vielleicht schwach positiv } \\
\text { schwach positiv }\end{array}$ & $\begin{array}{c}\text { ganz schwach negativ } \\
\text { neutral } \\
\text { positiv } \\
" \\
"\end{array}$ \\
\hline
\end{tabular}

Die im Kaolinplasma suspendierten B. K. bedürfen also einer deutlich größeren Konzentration an Lanthanionen zur Entladung, als die im Normalplasma suspendieren; die Blutkörperchen haben also im Kaolinplas ma positive Ladungen eingebüßt.

Eine ähnlich hemmende Wirkung auf die B. K.-Senkung wie die Ausschüttelung mit negativen Adsorbenzien bewirkt auch der $\mathrm{Zusatz}$ einer Lecithinemulsion zum Plasma. Es ist danach wahrscheinlich, daß auch das Lecithin seine Wirkung als Adsorbens für positive Teilchen ausübt.

Versuch vom 23. VII. 1919.

$$
\text { Lecithin. }
$$

0,6 Gravid.-Plasma

$0,10,95 \% \mathrm{NaCl}$

0,4 B. K.

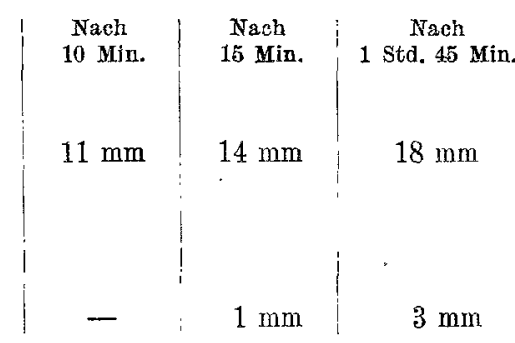

0,6 Gravid.-Plasma

0,1 Lecithinemulsion

$\{0,3$ Lecithin in Äther gelöst in $20 \mathrm{~cm} \mathrm{NaCl} \mathrm{ein-}$ geträufelt, Xther verdunstet)

0,4 B. K.

Unter den angeführten Gesichtspunkten ist wohl auch ein Verständnis für die öfter beobachtete Erscheinung zu gewinnen, daß die Sedimentierung der B.K. in isotonischer Kochsalzlösung im allgemeinen langsamer verläuft als im Plasma. Mit diesem Vorgang haben sich neuerdings wieder Berczeller und Sta $n k^{2} r^{1}$ ) sowie de Haan (l. c.) beschäftigt. Ihre Untersuchungen beziehen sich jedoch auf Tierblut; ich werde deshalb erst in meinér zweiten Mitteilung, die sich ebenfalls mit Untersuchungen an Tierblut beschäftigen wird, auf ihre Ergebnisse genauer eingehen.

Hier sei nur hervorgehoben, daß nach de Haan der enorme Unterschied in der Sedimentierungsgeschwindigkeit von Pferde- und RinderB. K. im Eigenplasma verschwindet, sobald man die B. K. in Kochsalzlösung aufschwemmt; beide sinken dann gleich langsam. Und Berczeller und Stanker, die mit B. K. von Rind und Schwein arbeiteten, fanden, daß die Senkung der B. K. bei steigenden Zusätzen von Kochsalzlösung mehr und mehr abnimmt, aber von einer bestimmten Plasmaverdünnung $a b$ dieser Abfall sprunghaft wird.

1) Internat. Zeitschr. f. Physikal.chem. Biolog. 3, 133. 1917. 
Nach unserer Auffassung des Sedimentierungsvorgangs könnte die Senkungsverzögerung durch Kochsalzlösung darauf beruhen, daß der elektropositive agglutinierende Plasmabestandteil, welcher der B. K.Oberfläche durch Adsorption anhaftet, in Kochsalzlösung die B. K.Oberfläche mehr oder weniger verläßt. Ist diese Auffassung zutreffend dann müssen die B.K. in Kochsalzlösung stärker negativ geladen sein als im Plasma. Das ist in der Tat der Fall, wie folgender Kataphoreseversuch beweist:

\begin{tabular}{|c|c|c|}
\hline \multirow{2}{*}{ La-Zusatz } & \multicolumn{2}{|c|}{ Ladung der B. K. nach Suspension in } \\
\hline & $\mathrm{NaCl}$ & Plasma \\
\hline $\mathrm{m} / 2500$ & negativ & schwach negativ \\
\hline $\mathrm{m} / 1500$ & $n$ & ganz schwach negativ \\
\hline$m / 1000$ & 19 & neutral \\
\hline $50 / 750$ & $"$ & positiv \\
\hline $\mathrm{T} / 260$ & 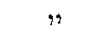 & $n$ \\
\hline $\mathrm{m} / 50$ & $n$ & $n$ \\
\hline${ }^{\mathrm{m}} / 10$ & neutral & $"$ \\
\hline
\end{tabular}

Die negative Aufladung der B. K. durch die Suspension in Kochsalzlösung ist also außerordentlich groß. Die zitierte Beobachtung von de Haan könnte danach zunächst so gedeutet werden, daß die verschiedene Sedimentierungsgeschwindigkeit der B. K. verschiedener Tiere in der Hauptsache von einem verschiedenen Gehalt der Plasmata an agglutinierender Substanz herrührt; wir werden in der 2. Mitteilung zeigen, daß dies jedoch nicht allgemein zutrifft. Die Beobachtung von Berczeller und Stanker läßt vielleicht darauf schließen, daß, entsprechend dem bekannten Verlauf der Adsorptionsisotherme im Gebiet der kleinen Plasmakonzentrationen der Einfluß auf die Ladung am größten ist; diese Hypothese wird experimentell noch zu prüfen sein.

Fragen wir nun weiter nach der chemischen Natur des im Plasma enthaltenen „Agglutinins", so liegt es jedenfalls nicht fern, in Erinnerung an die Immunitätsłreaktionen zu probieren, ob eine ,Hitzeinaktivierung" des Plasmaagglutinins möglich ist.

Ich brachte dazu das Plasma in ein Wasserbad von $56^{\circ} \mathrm{C}$ und belieB es unter genauer Einhaltung der Temperatur darin $1 / 2$ Stunde.

Versuch vom 12. VII. 1919.

0,8 Gravid.-Plasma unverändert

0,3 B. K. . . . . . . .

Nach 15 Min. Nach 30 Min.

$21 \mathrm{~mm}$
$5 \mathrm{~mm}$
0
0

Nach 3 Std.

$26 \mathrm{~mm}$

$25 \mathrm{~mm}$

$10 \mathrm{~mm}$

$7 \mathrm{~mm}$ 
In weiteren Versuchen ließ ich die Wärme verschieden lange Zeit auf das Plasma einwirken.

Versuch vom 21. 7. 1919.

0,6 Gravid.-Plasma bis $56^{\circ} \mathrm{O}$ erhitzt, dazu 0,2 B. K.

\begin{tabular}{c|c|c|c}
\hline $\begin{array}{c}\text { Dauer der } \\
\text { Hitzeeinwirkung }\end{array}$ & nach 12 Min, & $\begin{array}{c}\text { Sedimentierung } \\
\text { nach 25 Min. }\end{array}$ & nach 45 Min. \\
\hline $20 \mathrm{Min}$. & $16 \mathrm{~mm}$ & $20 \mathrm{~mm}$ & $20 \mathrm{~mm}$ \\
$30 \mathrm{Min}$. & $16 \mathrm{~mm}$ & $18 \mathrm{~mm}$ & $21 \mathrm{~mm}$ \\
$11 / 2 \mathrm{Std}$. & $11 \mathrm{~mm}$ & $18 \mathrm{~mm}$ & $17 \mathrm{~mm}$ \\
$3 \mathrm{Std}$. & $8 \mathrm{~mm}$ & $10 \mathrm{~mm}$ & $12 \mathrm{~mm}$
\end{tabular}

Es wird also in der Tat das Plasma durch Wärme inaktiviert, d. h. die Sedimentierung wird durch Erhitzung deutlich verzögert, und zwar um so stärker, je länger die Erwärmung dauert.

Nebenbei sei an dieser Stelle noch bemerkt, daß die Sedimentierung der B. K. dureh Abkühlung stark verzögert wird. Das zeigen etwa folgende Protokolle:

Blut einer Schwangeren.

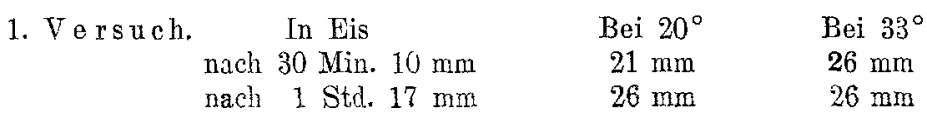

2. Versuch.

$\begin{array}{cc}\text { In Eis } & \text { Bei } 20^{\circ} \\ \text { nach } 20 \text { Min. } 0 & 14 \mathrm{~mm} \\ \text { nach } 40 \text { Min. } 0 & 20 \mathrm{~mm} \\ \text { Verbringen der Probe nach Umschütteln } \\ \text { In Temp. v. } 20^{\circ} & \text { In Eis } \\ \text { nach } 20 \text { Min. } 11 \mathrm{~mm} & 1 \mathrm{~mm} \\ \text { nach } 1 \text { Std. } 20 \mathrm{~mm} & 8 \mathrm{~mm}\end{array}$

Beim 2. Versuch wurde erst nach Abkühlung des Proberöhrchens das Blut eingefïlit und in Eis gestellt; nach 40 Min. war noch keine Spur von Senkung zu beobachten. Kam die Probe in Zimmertemp. von $20^{\circ}$, begann sofort eine intensive Senkung der B. K.

Ähnliche Beobachtungen über Temperatureinflüsse haben Fåhraeus und de Haan gemacht.

Durch diese Beobachtungen über den Einfluß erhöhter Temperatur wird unsere Aufmerksamkeit speziell auf die Eiweißkörper als mutmaßliche Agglutinine hingelenkt, und auch die folgenden Versuche können vielleicht als eine Stütze dieser Vermutung angesehen werden.

Während ich bisher nur Zitratblut verwendet hatte, machte ich nunmehr Kontrollversuche mit defibriniertem Blut und fand die zunächst überraschende Tatsache, daß die Defibrinier ung eine bedeutende Verzögerung der Sedimentierung erzeugt. 
Versuch vom 24. X. 1919.

Blut einer Gravida, in der ersten Probe in üblicher Weise mit 5\% Natr. citr. Lösung, in der 2. mit Hirudin $(0,8 \mathrm{mg}$ auf $5 \mathrm{ccm}$ Blut) versetzt und in der 3. durch Schlagen defibriniert.

$\begin{array}{cccc}\text { Serumhöhe } & \text { citratblut } & \text { Hirudinblut } & \text { Defibriniertes Blut } \\ 6 \mathrm{~mm} & \text { nach } 25 \mathrm{Min} . & \text { nach } 20 \mathrm{Min} . & \text { nach } 340 \mathrm{Min} . \\ 12 \mathrm{~mm} & \text { nach } 50 \mathrm{Min} . & \text { nach } 30 \mathrm{Min} . & \text { nach } 730 \mathrm{Min} . \\ 18 \mathrm{~mm} & \text { nach } 80 \mathrm{Min} . & \text { nach } 55 \mathrm{Min} . & \text { nach } 4200 \mathrm{Min} .\end{array}$

Der Unterschied zwischen defibriniertem Blut und den beiden anderen Proben ist gewaltig.

Die geringe Verzögerung des Citratblutes gegenüber dem Hirudinblut ist wohl durch die Verdünnung mit der Citratlösung bedingt.

Um zunächst auszuschließen, daß das Schlagen des Blutes an sich die Blutveränderung hervorruft, habe ich in einem weiteren Versuch Serum benutzt, das bei einem Aderlaß durch Spontangerinnung gewonnen war. Auch hier trat eine sehr große Verzögerung der Sedimentierung ein. Danach bleiben vor allem zwei Erklärungsmöglichkeiten zu erwägen:

Erstens kann das Fibrinogen selbst die senkungsbeschleunigende Substanz sein, und zweitens kann beim Gerinnen des Blutes das ,Agglutinin" mit entfernt, wegadsorbiert werden.

Für die erste Möglichkeit könnten die Erfahrungen sprechen, daß nach älteren Angaben in allen Zuständen, in denen eine Senkungsbeschleunigung eintritt, also besonders in der Gestation und in fieberhaften Krankheiten, auch eine Hyperinose, d. h. eine Vermehrung des Fibrins und damit natürlich auch des Fibrinogens statthat, andererseits in Blut, das sehr wenig Faserstoff enthält, wie das Nabelschnurblut, auch die Senkungsgeschwindigkeit stark herabgesetzt ist. Der große Unterschied in der Sedimentierungsgeschwindigkeit zwischen Nabelschnurblut und Blut der Gebärenden sei z. B. durch folgendes Beispiel dargetan:

\begin{tabular}{ccc}
\multicolumn{3}{c}{ Versuch v om 28. XT. 1919.} \\
Plasmahöhe von & Mutter & \multicolumn{2}{c}{ Kind } \\
$6 \mathrm{~mm}$ & nach $10 \mathrm{Min}$. & nach $195 \mathrm{Min}$. \\
$12 \mathrm{~mm}$ & nach $18 \mathrm{Min}$. & nach $475 \mathrm{Min}$. \\
$18 \mathrm{~mm}$ & nach $36 \mathrm{Min}$. & nach $1185 \mathrm{Min}$.
\end{tabular}

Dieser enorme Unterschied in der Stabilität steht in einem gewissen Verhältnis zu dem verschiedenen Fibrinogengehalt der beiden Blutarten.

Der Zusammenhang kann aber, wie gesagt, auch so gedeutet werden, daß das aus dem Fibrinogen entstehende Fibrin als Adsorbens auf die sedimentierende Substanz wirkt. Versuche, welche zur Entscheidung zwischen diesen Möglichkeiten beitragen, werden wir demnächst mit- 
teilen. Die Frage nach der chemischen Natur der sedimentierenden Substanz bleibt also noch offen.

Ich wende mich nun zu einer Reihe weiterer Versuche, durch Zusätze löslicher Stoffe zum Citratplasma die Sedimentierungsgeschwindigkeit künstlich zu ändern.

Da evtl. die Viscosität des Plasmas für das Fallen der B. K. von größerem Einfluß sein konnte, da vielleicht auch die Klebrigkeit von dem Plasma zugesetzten Substanzen im. Sinne der Gruberschen Agglutinationstheorie eine Rolle spielen konnte, wurde die Wirkung des $\mathrm{Zu}$ satzes von Gelatine, Agar, Gummi arabicum, isotonisch gemachtem Mundspeichel, Ovarialaysteninhalt und Stärkekleister untersucht.

Alle diese Substanzen haben eine stark beschleunigende Wirkung a uf die Senkung der B.K., und zwar nicht nur bei in Plasma, sondern auch bei in $\mathrm{NaCl}$ suspendierten gewaschenen $\mathrm{B} . \mathrm{K}$. Eine Anzahl von Protokollen soll dies illustrieren.

$$
\text { A. Gelatine. }
$$

Versuch vom 12. IX. 1919.

0,6 Blutplasma einer nicht graviden Frau, Naeh $10 \mathrm{Min}$, Nach 3 Std.

$0,110 \%$ in $\mathrm{NaCl} g$ elöste Gelatine

0,4 B. K. . . . . . . . . $22 \mathrm{~mm}$

$24 \mathrm{mrli}$

0,6 Blutplasma

$0,10,95 \% \mathrm{NaCl}$

0,4 B. K.

$0 \mathrm{~mm}$

$6 \mathrm{~mm}$

$0,60,95 \% \mathrm{NaCl}$

$0,110 \%$ Gelatine

0,4 B. K.

$16 \mathrm{~mm}$

$20 \mathrm{~mm}$

$0,60,95 \% \mathrm{NaCl}$

0,4 B. K.

$0 \mathrm{~mm}$

$4 \mathrm{~mm}$

Versuch rom 7. VIII. 1919.

0,9 Nabelschnurblut

$0,10,95 \% \mathrm{NaCl}$.

0,9 Nabelschnurblut

$0,15 \%$ Gelatine :

0,8 Nabelschnurblut

$0,20,5 \%$ Gelatine.

0,9 Nabelschnurblut

$0,10,5 \%$ Gelatine.

\begin{tabular}{|c|c|c} 
Nach $10 \mathrm{Min}$. & Nach $30 \mathrm{Min}$. & Nach 3 std. \\
$0 \mathrm{~mm}$ & $.2 \mathrm{~mm}$ & $3 \mathrm{~mm}$ \\
$8 \mathrm{~mm}$ & $15 \mathrm{~mm}$ & $22 \mathrm{~mm}$ \\
$4 \mathrm{~mm}$ & $7 \mathrm{~mm}$ & $13 \mathrm{~mm}$ \\
$2 \mathrm{~mm}$ & $4 \mathrm{~mm}$ & $7 \mathrm{~mm}$
\end{tabular}

Versuch vom 2. X. 1919.

\begin{tabular}{l|c|c}
0,6 Gravid.-Plasma & Nach 10 Min. & Nach 1 std. \\
$0,10,95 \%$ NaCl & & \\
0,4 B. K. . . . & $8 \mathrm{~mm}$ & $18 \mathrm{~mm}$ \\
0,6 Gravid.-Plasma & & \\
$0,15 \%$ Gelatine & $19 \mathrm{~mm}$ & $25 \mathrm{~mm}$ \\
0,4 B. K. . . . & $19 \mathrm{~m}$
\end{tabular}


Untersuchungen uber die Senkungsgeschwindigkeit der roten Blutkörperchen. 179

Es tritt also im Normalfrauenblut, im langsam sedimentierenden Nabelschnurblut, im schnell sedimentierenden Gravidenblut und in einer Suspension der B. K. in Kochsalzlösung eine gleichermaßen starke Senkungsbeschleunigung durch Gelatinezusatz ein; die Beschleunigung ist bis $0,05 \%$ noch deutlich.

B. Agar.

Versuch vom 8. IX. 1919.

\begin{tabular}{|c|c|c|c|c|}
\hline \multirow{3}{*}{$\begin{array}{ll}0,6 & \text { Ascitesserum } \\
0,1 & \mathrm{NaCl} \\
0 & \mathrm{R} K\end{array}$} & Nach 30 Min. & Nach 3 Std. & Nach 6 std. & \multirow{4}{*}{$\begin{array}{l}\text { Beobachtung } \\
\text { im Wasserbad } \\
\text { bei } 40^{\circ} \mathrm{C}\end{array}$} \\
\hline & & & & \\
\hline & $2 \mathrm{~mm}$ & $\bar{\jmath} \mathrm{mm}$ & $8 \mathrm{~mm}$ & \\
\hline $\begin{array}{l}0,6 \text { Ascitesserum } \\
0,11 / 2 \% \text { Agar } \\
0,4 \text { B. K. . . . }\end{array}$ & $4 \mathrm{mIm}$ & $19 \mathrm{~mm}$ & $20 \mathrm{~mm}$ & \\
\hline
\end{tabular}

Versuch vom 14. IX. 1919.

0,6 Normalplasma

$0,10,95 \% \mathrm{NaCl}$

0,4 B. K.

Nach 3 Std.

0,6 Normalplasma

$0,1 \quad 10 \%$ Grummi arabicum

0,4 B. K. . . . . .

$0,70,95 \% \mathrm{NaCl}$

0,4 B. K

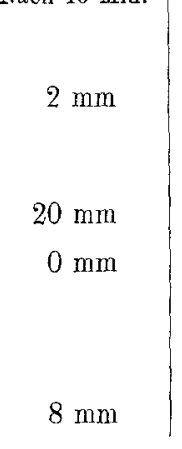

Nach 20 Min.

Nach 3 std.

$0,6 \quad 0,95 \% \mathrm{NaCl}$

$0,1 \quad 10 \%$ Gummi arabicum

0,5 B. K.

D. Speichel.

Versuch vom 3. IX. 1919.

0,6 Gravid.-Plasma

$0,10,95 \% \mathrm{NaCl}$

0,4 B. K.

\begin{tabular}{|c|c|c} 
Nach 30 Min. & Nach 1 Std. & Nach 2 Std. \\
$8 \mathrm{~mm}$ & $13 \mathrm{~mm}$ & $15 \mathrm{~mm}$ \\
$12 \mathrm{~mm}$ & $16 \mathrm{~mm}$ & $16 \mathrm{~mm}$
\end{tabular}

0,6 Gravid.-Plasma

0,1 Mundspeichel mit $\mathrm{NaCl}$ isotonisch gemacht

0,4 B. K

E. Ovarialcysteninhalt.

Versuch vom 25. VIII. 1919.

0,9 Gravid.-Blut

$0,9 \quad 0,95 \% \quad \mathrm{NaC}$

Nach 30 Min.

0,9 Gravid.-Blut

0,9 schleimiger Inhalt einer Ovarialcyste

$2 \mathrm{~mm}$

$20 \mathrm{~mm}$ 
Versuch rom 11. IX. 1919.

$0,80,95 \%$ NaCl
0,4 B. K. . .
$0,60,95 \%$ NaCl
0,2 Cysteninhalt
0,4 B. K. . . .

\begin{tabular}{|c|c} 
Nach 30 Min. & Nach 4ã Min. \\
$1 \mathrm{~mm}$ & $2 \mathrm{~mm}$ \\
$14 \mathrm{~mm}$ & $18 \mathrm{~mm}$
\end{tabular}

F. Stärkekleister.

Versuch vom 17. IX. 1919.

0,6 Gravid.-Plasma

$0,10,95 \% \mathrm{NaCl}$

0,4 B. K.

\begin{tabular}{|c|c|c|}
\hline Nach 20 Min. & Nach 45 Min. & Nach 1 std. \\
\hline $7 \mathrm{~mm}$ & $14 \mathrm{~mm}$ & $20 \mathrm{~mm}$ \\
\hline $15 \mathrm{~mm}$ & $20 \mathrm{~mm}$ & $21 \mathrm{~mm}$ \\
\hline
\end{tabular}

0,6 Gravid.-Plasma

$0,12,5 \%$ Stärkekleister in $\mathrm{NaCl}$

0,4 B.K. . . . . . . .

Außerdem habe ich Zusätze von verschieden prozentigen Lösungen
w. Suspensionen von Dextrin, von Inulin und Cholesterin versucht, dabei aber keine eindeutigen Resultate erhalten.

Wie soll man die senkungsbeschleunigte Wirkung der viscösen Stoffe nun beurteilen? Zunächst würde man ja wohl annehmen, daß derartige Körper, welche die innere Reibung des Suspensionsmittels erhöhen, die Senkung der B. K. verzögern. Aber das Gegenteil tritt ein, und der Grund ist wiederum die verstärkte Agglutination. Schon makroskopisch läßt sich diese nach Zusatz der klebrigen Körper als Krümelbildung feststellen. Es ist also wieder die starke Oberflächenverkleinerung, die die Senkungsbeschleunigung bedingt. Ob dabei aber eine Verklebung der Zellen im Sinne der Gruberschen Hypothese wesentlich ist, ist auf Grund von Kataphoreseversuchen $z u$ bezweifeln. Diese lehren. nämlich, daß durch Gelatinezusatz die elektro-negativen B. K. mehr oder weniger entladen werden, wie das folgende Protokoll zeigt:

Vergleich 1. 0,4 Gravid.-B. K. $+0,6$ Gravid.-Plasma + 0,1 NaCl mit 2. 0,4 Gravid.-B. K. + 0,6 Gravid.-Plasma + 0,1 5\% Gelatine NaCl.

\begin{tabular}{c|c|c}
\hline La.Zusatz & Gewöhnl. Plasma & Gelatineplasma \\
\hline $\mathrm{m} / 2500$ & negativ & schwach negativ \\
$\mathrm{In} / 1500$ & schwach negativ & neutral \\
$\mathrm{In} / 1000$ & neutral & schwach positiv \\
$\mathrm{m} / / 50$ & neutral & positiv \\
$\mathrm{In} / 500$ & positiv & positiv
\end{tabular}

Worauf die entladende Eigenschaft der Gelatine beruht, bleibt noch zu untersuchen.

Die senkungsbeschleunigende Wirkung klebriger Stoffe war übrigens schon den älteren Hämatologen wie Lehmann, Nasse, Johannes Müller wohlbe- 
kannt. Auch in neuerer Zeit sind von $\left.\mathrm{Brat}^{1}\right), \mathrm{Biernacki}^{2}$ ) u. a. hierher gehörige Beobachtungen veröffentlieht worden.

Sack ur ${ }^{3}$ ) studierte den EinfluB am hängenden Tropfen; er fand, daB Zusatz von Gelatine zu.Blut folgende Veränderungen im Gesichtsfeld hervorruft: Die herumschwimmenden roten $B$. K. nähern sich einander und legen sich schnell fest zusammen. Indem jedes freischwimmende B. K., sobald es an einen derartigen Komplex von Erythrocyten herangerät, festgehalten wird, häufig sogar direkt herangezogen zu werden scheint, bilden sich teils Geldrollen, teils Schollen, die sich durch gegenseitige Annäherung in einem Bruchteil einer Minute zu einem netzförmigen Gerüste zsammenlegen.

Zu den löslichen Substanzen, durch deren Zusatz zum Blut man die Sedimentierungsgeschwindigkeit der roten B. K. künstlich verändern kann, gehören auch die Narkotica.

Diese wurden in Konzentrationen verwendet, welche nach den Versuchèn von $\mathrm{Joel}^{4}$ ) aus dem hiesigen physiologischen Institut die Permeabilität der B. K. für Elektrolyte noch nicht steigern, also sicher nicht Hämolyse hervorrufen. Es wurden in isotonischer NaCl-Lösung gelöst verwendet:

$.0,9 \%$ Amylalkohol, $0,7 \%$ Phenylharnstoff, $1,2 \%$ Isobutylurethan, $0,05 \%$ Acetophenon und $0,23 \%$ Heptylalkohol; diese Lösungen wurden dann im Versuch mit Blut auf das 5-10fache' verdünnt.

Es stellte sich heraus, daß alle verwendeten Narkotica eine hemmende Wirkung a ufdas Fallen der K. B. a usüben, wie die folgenden Protokolle zeigen:

Versuch $\nabla$ om 12. IX. 1919

0,5 Gravid.-Plasma

$0,20,95 \% \mathrm{NaCl}$

0,3 B. K.

0,5 Gravid.-Plasma

$0,20,9 \%$ Amylalkohol

0,3 K. B.

\begin{tabular}{|c|c|c} 
Nach 30 Min. & Nach 1 Stã. & Nach 2 Std. \\
$8 \mathrm{~mm}$ & $14 \mathrm{~mm}$ & $18 \mathrm{~mm}$ \\
$6 \mathrm{~mm}$ & $10 \mathrm{~mm}$ & $16 \mathrm{~mm}$
\end{tabular}

Versuch vom 10. IX. 1919.

1,0 Gravid.-Plasma

$0,4 \quad 0,95 \% \mathrm{NaCl}$

0,4 B. $\mathrm{K}$.

1,0 Gravid.-Plasma

$0,4 \quad 0,7 \%$ Phenylharnstoff

0,4 B. K.

Nach 15 Min.
$7 \mathrm{~mm}$
$3 \mathrm{~mm}$

Nach 30 Min.

$18 \mathrm{~mm}$

$7 \mathrm{~mm}$
Nach 50 Min.

$21 \mathrm{~mm}$

$10 \mathrm{~mm}$

1) Zeitschr. f. klin. Medizin 56, 380. 1905.

2) Zeitschr. f. physiol. Chemie 19, 179. 1894 und Zeitschr. f. klin. Medizin 24, 460. 1896.

3) Grenzgebiete d. Medizin u. Chirurgie 8, 188.

4) Arch. f. d. ges. Physiol. 161. 1915. 
Versuch vom 12. IX. 1919

0,5 Gravid.-Plasma

$0,20,95 \% \mathrm{NaCl}$

0,3 B. K.

0,5 Gravid.-Plasma

0,2 Isobuthylurethan $1,2 \%$

0,3 B. K.

\begin{tabular}{|c|c|c|c|c}
20 & $30^{\circ}$ & $1 \mathrm{Uhr}$ & $3 \mathrm{Uhr}$ \\
6 & 8 & 16 & 19 \\
3 & 4 & 11 & 16
\end{tabular}

Von der Natur dieser Wirkung der Narkotica haben wir uns folgendes Bild gemacht:

Als Stoffe von großer Oberflächenaktivität reichern sich die Narkotica an der Blutkörperchenoberfläche an und verdrängen dabei den agglutinierenden Körper. Mit dieser Erklärung steht die Beobachtung in Übereinstimmung, daß sich eine durch Gummi arabicum hervorgerufene Senkungsbeschleunigung durch Narkotica wieder aufheben läßt, z. B.:

Versuch vom 14. IX. 1919.

\begin{tabular}{|c|c|c|}
\hline $\begin{array}{l}0,7 \mathrm{NaCl} 0,95 \% \\
0,15 \% \text { Gummi arabicum } \\
0,4 \text { B. K. . . . . . . }\end{array}$ & Nach 15 Min. & Nach $2^{2 / 2}$ Std \\
\hline $\begin{array}{l}0,60,95 \% \mathrm{NaCl} \\
0,15 \% \text { Gummi arabicum } \\
0,10,7 \% \text { Phenylharnstoff } \\
0,4 \text { B. K. . . . . . . . }\end{array}$ & $2 \mathrm{~mm}$ & $14 \mathrm{~mm}$ \\
\hline
\end{tabular}

Ich breche an dieser Stelle die Darlegung meiner Versuchsergebnisse vorläufig $a b$ und behalte mir die Fortsetzung in einer 2. Mitteilung vor. Es hat sich nämlich gezeigt, daß sich das Blut mancher Tiere dem Anschein nach wesentlich anders verhält als das Menschenblut; die vorliegende Mitteilung faßt deshalb zunächst bloß unsere Erfahrungen an Menschenblut zusammen.

\section{Zusammenfassung.}

1. Die beschleunigte Sedimentierung und verstärkte Hämagglutination im Gravidblut ist abhängig von einer relativen Entladung der negativ geladenen Blutkörperchen durch einen bei der Gravidität im Blutplasma auftretenden, vermutlich positiv geladenen Körper. Dies wird bestätigt durch den Ausfall von Adsorptionsversuchen mit Adsorbenzien für positive bzw. negative Teilchen. Nach Ausschüttelung des Plasma mit Tierkohle, Kaolin, Bolus alba und in geringerem Maße mit Talkum, d. h. mit Stoffen, welche vornehmlich positive Teilchen adsorbieren, wird die Suspensionsstabilität des Blutes erhöht und die negative Ladung der B. K. verstärkt, während Aluminiumhydroxyd, Cerioxyd, Eisenhydroxyd, welche vorwiegend negative Teilchen festhalten, die Senkungsgeschwindigkeit nicht verändern (oder vorübergehend vergrößern). 
Untersuchungen ïber die Senkungsgeschwindigkeit der roten Blatkörperchen. 183

2. Auch durch Erwärmen läßt sich das Plasma derartig verändern (inaktivieren), daß die Senkungsbeschleunigung gehemmt wird.

3. Durch die Gerinnung, also durch die Entfernung des Fibrins aus dem Plasma, wird die Senkungsbeschleunigung aufgehoben. Ob das Fibrinogen selbst der senkungsbeschleunigende Körper ist, oder ob mit der Bildung des Fibrins der fragliche Körper aus dem Plasma mitentfernt vielleicht adsorbiert wird, muß noch weiter untersucht werden.

4. Klebrige Stoffe, wie Gelatine, Gummi arabicum, Mucin u. a. steigern die Sedimentierungsgeschwindigkeit. Dabei werden die B. K. mehr oder weniger entladen.

5. Narkotica hemmen die B. K.-Senkung, wahrscheinlich dadurch, daß sie die agglutinierende Substanz des Plasmas von der B. K.-Oberfläche verdrängen. 